\title{
The first glimpse determines the perception of an ambiguous figure
}

\author{
GARVIN CHASTAIN and CLARKE A. BURNHAM \\ University of Texas, Austin, Texas 78712
}

\begin{abstract}
The ambiguous rat-man figure was tachistoscopically presented to 36 subjects in successive segments to test the hypothesis that the starting segment would determine the perception of the figure. Starting segments were selected which were expected to produce the perception of a rat, a man, or either a rat or man. The remaining segments came from figures evaluated in a preliminary study. The selected figures differed in drawn bias and tended to be seen as a rat, a man, or either a rat or a man. The three starting segments were combined factorially with the three levels of drawn bias of the remaining segments. The effect of the starting segment was significant; the effect of drawn bias was not. A further experiment showed that presentation of the rat vs. man starting segments by themselves did not produce a reliable difference. The results support a constructive model of form perception in which the stimulus material first presented establishes a hypothesis which is used to interpret the remaining material.
\end{abstract}

Constructive models of form perception converge on the notion that the recognition process involves the formation and testing of hypotheses about the identity of the stimulus pattern. One source of the perceiver's initial hypothesis is his set or expectation. Expectation manipulations have been shown to affect the perception of ambiguous stimuli (e.g., Bruner \& Minturn, 1955; Bugelski \& Alampay, 1961). Another possible source of the initial hypothesis, investigated in this study, is the segment of the figure which is first fixated or glimpsed. A perceiver may form a tentative hypothesis about the identity of a figure from the information contained in the segment which is first glimpsed; he may then attempt to interpret subsequent glimpses in a manner consistent with this hypothesis. If successful, his perceptual hypothesis will be confirmed and the figure will be recognized.

The hypothesis that the first glimpse determines recognition was tested by presenting the ambiguous rat-man drawing (Figure 1) to subjects in sequential segments and manipulating the segment shown first. The segment used to induce a "man" hypothesis was Segment 1 , the portion of the drawing containing the upper part of the nose and the eyes. This was selected because of findings such as Howells's (1938), that the area above the middle of the nose has the most value for facial recognition. The segment used to induce a "rat" hypothesis was Segment 5, the portion of the drawing containing what is probably the most distinctive feature of a rat, its long and rather unique tail. Segment 6 of the drawing was also presented first; it was expected that this segment would not selectively favor either the rat or the man hypothesis. Following the initial presentation of one of these

Alfred McAlister physically and materially assisted in the preparation of the film segments.

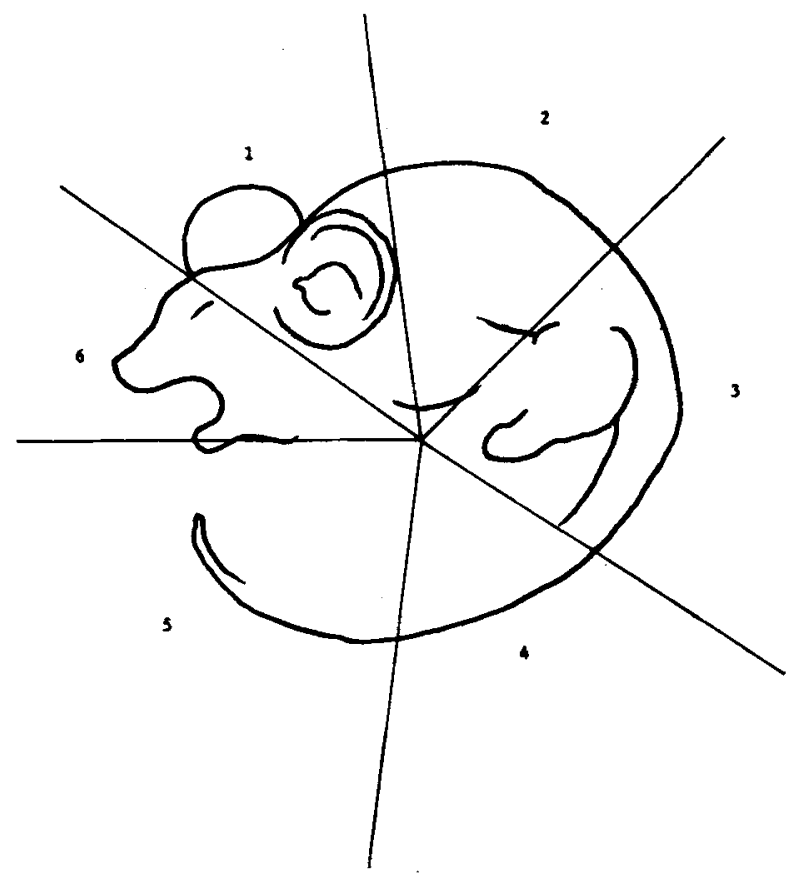

Figure 1. The unblased rat-man figure with segments indicated.

segments, the remaining five segments were shown successively in either a clockwise or a counterclockwise order.

Ambiguous figures can be drawn so that one of the alternative perceptions is more probable when the drawing is normally viewed (Fisher, 1967). If, however, the starting segment of a sequentially presented figure determines the perceiver's initial hypothesis about the nature of the figure, moderate amounts of drawn bias should have no effect on the resulting perception. The later segments of such 
figures could be assimilated to the hypothesis generated by the initial segment. Drawn bias was included as a variable in order to evaluate the strength of the effect of the starting segment.

The sequential presentation experiment, which combined factorially the variables of starting segment and drawn bias, was preceded by a drawing selection experiment. The sequential presentation experiment was followed by a supplementary experiment in which only the starting segments were presented.

\section{EXPERIMENT I: DRAWING SELECTION}

The purpose of this experiment was to select rat-man drawings which differed in drawn bias for use as stimuli in the sequential presentation experiment. Three drawings were desired-one which tended to be recognized as a man, one which tended to be recognized as a rat, and one which was unbiased toward either interpretation.

\section{Method}

An artist prepared nine drawings of the rat-man figure. Four increasingly emphasized man features, four increasingly emphasized rat features, and one was presumed neutral. The subjects in this and all subsequent experiments were students fulfilling a requirement of the introductory psychology course. Eighteen subjects participated in Experiment $I$ in two groups of nine. They were told that they were going to view a number of figures and that some would look more like a man and some more like a rat. They made their responses on a numbered sheet and were limited to rat and man designations. Slides of the drawings were projected on a screen with the image subtending a visual angle of $4^{\circ}$. The stimulus duration was $5 \mathrm{sec}$; the subjects were told to respond within that interval. Each of the nine drawings was presented three times; the order of the stimuli was random within each block of the nine drawings.

\section{Results}

The data were analyzed by plotting the percentage of rat responses for the nine levels of drawn bias. The percentage of rat responses increased with drawn bias and reached an asymptote of approximately $95 \%$ for the three most rat-like drawings. Those drawings identified as a rat $14.8 \%, 35.2 \%$, and $70.4 \%$ of the time were selected for the sequential presentation experiment. These drawings were closest to the 25th, 50 th, and 75 th percentiles. The rat-biased and manbiased figures are shown in Figure 2.
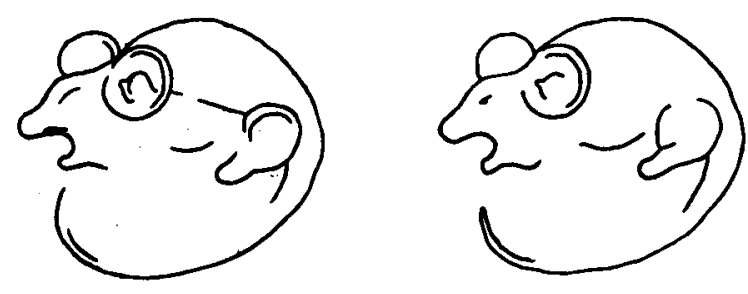

Fig. 2. The man (left) and rat (right) figures.

\section{EXPERIMENT II: SEQUENTIAL PRESENTATION}

The hypothesis that the first glimpse of an ambiguous figure would determine its perception was tested by presenting the rat-man figure in successive segments, starting with one of the segments expected to arouse a rat hypothesis, a man hypothesis, or either a rat or a man hypothesis. The starting segment was always taken from the most ambiguous figure. The segments presented after the starting segment came from the figures which had previously been found to be usually recognized as a rat, a man, or either a rat or a man. It was expected that the starting segment would affect the perception and that the drawn bias of the remaining segments would not.

\section{Method}

The three levels of drawn bias were combined factorially with the three starting segments to generate nine experimental conditions. In each, the rat-man figure was presented in successive segments, starting with the selected segment of the most ambiguous figure and continuing with the remaining segments from the figures differing in drawn bias. Each condition was presented in two versions. In one, the figure was built up in a clockwise direction from the starting segment; in the other, it progressed in a counterclockwise direction. The segments always appeared in their proper respective positions in the full figure, and the lines in adjacent segments were continuous.

The stimuli sequences were prepared by photographing the segments of the drawings on a white ground on $8-\mathrm{mm}$ film. Thus the lines in each segment were perceived as dark contours on a full-screen white background and the presentation of a successive segment served to mask the preceding segment by luminance summation. The final segment of the figure was followed by a segment containing the phrase, "WHICH ONE?" This was included to provide a mask for the last segment of the figure. The subjects were told that this phrase would end the presentation.

The film was projected in an illuminated room onto a $15.3 \mathrm{x}$ $20.3 \mathrm{~mm}$ white screen with a black fixation point at its center. The entire figure subtended a visual angle of $4^{\circ}$. The projector was run at a speed of 54 frames/sec; each segment was on 3 frames. Hence, each segment was viewed for $55.6 \mathrm{msec}$ and the entire figure was presented in $334 \mathrm{msec}$.

Thirty-six introductory psychology students participated in the study. Each subject was run individually and received only one trial on the rat-man figure. They were randomly assigned to one of the nine experimental conditions, with two of the four subjects in each condition experiencing the clockwise sequences and two the counterclockwise sequences. The subjects were told to look at the fixation point and that they would experience a brief presentation of a figure in sequential parts-"as if the figure were cut into pieces and shown, one piece at a time, in its respective position."

Two geometric figures, a circle and a square, were first shown and the subject was asked to verbally identify the figure. Then the rat-man figure was shown and the subject was asked to check on a response sheet the term which best corresponded to his perception. The subject did not receive the response sheet until after the figure was presented. The possible responses were: Man, Man with a few ratlike characteristics, Man with many ratlike characteristics, Rat with many manlike characteristics, Rat with a few manlike characteristics, and Rat.

\section{Results}

The responses were assigned numerical scores from 6 (man) to 1 (rat), and the data were analyzed by a three-way analysis of variance. The effect of the 
starting segment was significant $(F=5.56$, df $=$ $2 / 18, p<.02$ ). The mean ratings for the three levels of starting segment were: man segment, 3.58 ; neutral segment, 2.50; and rat segment, 1.75. The distributions of responses for the man and rat starting segments were skewed with modes of 5 and 1 , respectively. The distribution of responses for the neutral starting segment was bimodal. The effect of drawn bias was not significant $(F=2.25$, $\mathrm{df}=2 / 18)$. Neither the effect of order (clockwise vs. counterclockwise presentation) nor the interactions approached significance (all ps $>.30$ ).

The hypothesis that the first glimpse of an ambiguous figure determines the perception of that figure was thus confirmed. The results indicate that subsequent glimpses of figures differing in drawn bias do not consistently influence perception.

\section{EXPERIMENT III: STARTING SEGMENT ALONE}

The results of the sequential presentation study are consistent with the introductory statements that the recognition process is a hypothesis plus interpretation process. However, the results are also consistent with the hypothesis that the response differences depend solely on the information contained in the starting segment and that the remainder of the figure is irrelevant for the resulting perception. That is, the subjects may have been able to determine whether the figure was a rat or a man solely from the information in the starting segment. If so, the sequential presentation method would not test the proposed constructive model of form perception. This model implies that the initial information is used to create a hypothesis which is then used to interpret the remaining input, and that this remaining input is essential to produce a stable perceptual effect. Therefore, an additional experiment was conducted in which subjects were presented with only the rat or man starting segments under viewing conditions similar to those used in the preceding experiment. The subjects were asked to report what the entire figure would have been if all of it had been shown. No significant difference between the rat and man starting segments was expected in this starting segment alone experiment.

\section{Method}

The man and the rat starting segments of the neutral figure were copied onto cards for tachistoscopic presentation. They were presented for $55.6 \mathrm{msec}$, which was the duration of the segments in the sequential presentation study, and preceded and followed by a light field with a fixation point. The subjects, 24 introductory psychology students, were run individually. Half were shown the man starting segment and half the rat starting segment. The subject was first shown two geometric practice figures at the exposure duration of $55.6 \mathrm{msec}$. He was then told that the next figure would be part of a mammal. After the presentation of the starting segment, he was shown the response sheet used in the sequential presentation study and asked to answer it according to his judgment of what the entire figure would have been if all of it had been shown.

\section{Results}

The responses were assigned numerical scores from 6 (man) to 1 (rat), and these data were analyzed by a one-way analysis of variance. The effect of the segment did not approach significance $(F<1.0)$, although the means were in the direction of the differences obtained in the sequential presentation study. The mean for the rat starting segment was 1.5 , and the mean for the man starting segment was 2.0 .

- Hence, the information in the man and rat starting segments is not, in itself, sufficient to produce a reliable difference. The effect of presenting the other segments with the starting segments may be evaluated by subtracting the starting segment difference of .5 from the difference of 1.83 produced by the man vs. rat starting segments in the sequential presentation study. A one-way analysis of variance contrasting the subjects in the sequential presentation study who received the rat vs. man start showed that the reduced difference of 1.33 was statistically significant $(\mathrm{F}=$ 6.68 , df $=1 / 22, \mathrm{p}<.02$ ). The different starting segments apparently must be combined with other information to generate a reliable perceptual difference.

\section{DISCUSSION}

The results of these experiments indicate that: (1) the perception of sequentially presented ambiguous figures can be affected by controlling the segment which is first viewed; (2) it is necessary to follow the first segment with some additional segments to produce this effect; and (3) the drawn bias of the remaining segments does not influence the resulting perception. These conclusions must, of course, be limited both to the particular stimulus used in these studies, the rat-man figure, and to the methodology of brief exposure durations.

The results support a constructive model of form perception. The perceiver creates a hypothesis from an analysis of the segment which is presented first. He then attempts to interpret the remaining segments in terms of this initial hypothesis. The single segment study showed that stimuli which create different initial hypotheses do not, by themselves, produce reliably different perceptual effects. The different starting segments must be followed by additional segments for the constructive processes to flesh out perceptually different mammals. The starting segments initiate a hypothesis, plan, blueprint, or schema (Hochberg, 1968; Neisser, 1967) to guide the subsequent constructive processes.

Our interpretation of these findings is, of course, tentative. It may also appear paradoxical. How can the starting segment affect the perception of the remaining segments if it, by itself, cannot be reliably categorized as part of a rat or part of a man? The subjects in the starting-segment-alone experiment were required to make an inference from a minimum 
amount of information. They were told that the presented segment would be part of a mammal and either Segment 1 or Segment 5 of Figure 1 was shown briefly. They were then asked to decide where on the rat-man response scale the complete figure would have been had all of it been presented. Perhaps the subjects presented with the rat segment (Segment 5) saw this segment as part of a body and the subjects presented with the man segment (Segment 1) saw a portion of a face. Neither of these two percepts would, by themselves, produce a perception of a rat or a man. In fact, both could lead to the inference that the entire figure would have been an animal. This inference would account for the fact that the responses in the starting segment alone study were near the rat end of the response scale.

These initial perceptions could generate hypotheses which would organize the perception of the remaining segments so that subjects would report seeing an integrated picture which was either the profile of a rat or the face of a man. The perception of a rat could occur by the application of the hypothesis "part of a body" to the remaining segments. Similarly, the perception of a man's face could arise from the application of a hypothesis such as "part of a face." The organizing hypothesis produced by the neutral starting segment, Segment 6 of Figure 1, apparently was equivocal with respect to these perceptions.

This interpretation of the results also accounts for the lack of an effect of drawn bias in the sequential presentation study. The bias was not sufficient to override the effect of the initial hypotheses; the remaining segments could be assimilated into either the face or body organizational schema. It is likely that drawn bias would have producesd an effect if the exposure durations had been longer or if the bias had been more extreme. The short exposure conditions could have precluded a critical evaluation of the initial hypotheses; more extreme bias could have led to its disconfirmation. Studies such as Bruner and Potter's (1964), which demonstrated that initially incorrect hypotheses hinder recognition, have used both longer exposures and pictures which generate initial hypotheses which are quite discrepant from the presented stimuli.

It is possible that many of the perceptual set or expectation effects reported in the literature are mediated by initial fixations. Those subjects in Bugelski and Alampay's (1961) study who had been exposed to unambiguous animal pictures and subsequently saw the rat-man figure as a rat may have initially looked at a different segment of the picture than those who had either been previously exposed to humans or saw no set-inducing figures. The lack of a set effect for verbal descriptions in Leeper's (1935). study of the ambiguous wife/mother-in-law drawing may have occurred because verbal descriptions do not reliably control initial fixations for this figure. A set effect was obtained with the prior presentation of an: unambiguous figure. Previous viewing of an unambiguous figure would be expected to control the initial fixation of a similar ambiguous figure.

\section{REFERENCES}

Bruner, J. S., \& Potter, M. C. Interference in visual recognition. Science, 1964, 144, 424-425.

Bruner, J. S., \& Minturn, A. L. Perceptual identification and perceptual organization. Joumal of General Psychology, 1955, 53, 21-28.

Bugelski, B. R., \& Alampay, D. A. The role of frequency in developing perceptual sets. Canadian Journal of Psychology, 191, 15, 205-211.

Fisher, G. H. Preparation of ambiguous stimulus materials. Perception \& Psychophysics, 1967, 2, 421.422.

HochaERG, J. In the mind's eye. In R. N. Haber (Ed.), Contemporary theory and research in visual perception. New York: Holt, Rinehart and Winston, 1968. Pp. 303-331.

Howells, T. J. A study of ability to recognize faces. Journal of Abnormal Psychology, 1938, 33, 124-127.

LEEPER, R. A study of a neglected portion of the field of learning: The development of sensory organization. Journal of Genetic Psychology, 1935, 46, 41-75.

NeIsser, U. Cognitive psychology. New York: Meredith, 1967.

(Received for publication April 4, 1974; revision received September 13, 1974.) 